\title{
Characterisation of Respiratory Motion Extracted from 4D MRI
}

\author{
Ashrani Aizzuddin Abd. Rahni ${ }^{b, a}$, Emma Lewis ${ }^{a}$ and Kevin Wells ${ }^{a}$ \\ ${ }^{a}$ Centre for Vision, Speech and Signal Processing, University of Surrey, Guildford, GU2 7XH; \\ ${ }^{b}$ Universiti Kebangsaan Malaysia, Malaysia
}

\begin{abstract}
Nuclear Medicine (NM) imaging is currently the most sensitive approach for functional imaging of the human body. However, in order to achieve high-resolution imaging, one of the factors degrading the detail or apparent resolution in the reconstructed image, namely respiratory motion, has to be overcome. All respiratory motion correction approaches depend on some assumption or estimate of respiratory motion. In this paper, the respiratory motion found from $4 \mathrm{D} \mathrm{MRI}$ is analysed and characterised. The characteristics found are compared with previous studies and will be incorporated into the process of estimating respiratory motion.
\end{abstract}

Keywords: Nuclear Medicine, Respiratory motion, Affine Transformation, Principal Component Analysis, Hysteresis, 4D MRI

\section{INTRODUCTION}

Compensation of respiratory motion has been identified as an important factor in achieving high resolution NM imaging. Respiratory gating is seen as an attractive compensation approach due to its simplicity. However, gated images do not take advantage of all acquired data and hence have a higher SNR. ${ }^{1}$ On the other hand all motion correction approaches depend on some assumption or estimate of respiratory motion. Obtaining respiratory motion information from the NM acquired data itself imposes the low spatial resolution of NM imaging on the correction process. ${ }^{2}$ Alternatively, the respiratory motion information found using an additional dynamic volumetric imaging modality may not be directly applicable for motion correction during NM image acquisition. ${ }^{3}$ Therefore, this paper follows the approach of using an external source or surrogate of respiratory motion to infer internal respiratory motion.

The approach chosen follows previous work where the external source of respiratory motion is proposed to be a stereo camera capture of the anterior surface of the torso. Respiratory motion is then estimated using recursive Bayesian estimation, in the form of particle filtering, as proposed in the previous study using the XCAT phantom. ${ }^{4}$ However, in this study, $4 \mathrm{D}$ medical imaging data is used as the source of respiratory motion. This motion is found via organ-wise affine registration as previously performed with the XCAT phantom. ${ }^{5}$ To make motion estimation more accurate, the characteristics of external and internal motion found from the 4D medical imaging dataset is analysed in this paper.

\section{ANALYSIS AND RESULTS}

The MRI data were acquired using a Siemens MAGNETOM $®$ Verio 3T MRI scanner. The dynamic volumes have an in-/plane resolution of $1.534 \mathrm{~mm}$ and slice separation of $5 \mathrm{~mm}$. The slices were acquired in the coronal plane. The volumes were acquired every $0.956 \mathrm{~s}$ in two sets of sequences of 10 volumes each, which are acquired 17 minutes apart from one another. The method of organ-wise affine registration is described in (Rahni et al., 2013). ${ }^{6}$

Medical Imaging 2013: Image Processing, edited by Sebastien Ourselin, David R. Haynor,

Proc. of SPIE Vol. 8669, 86692Z · @ 2013 SPIE · CCC code: 1605-7422/13/\$18

doi: $10.1117 / 12.2006859$

Proc. of SPIE Vol. 8669 86692Z-1 


\subsection{Organ Configuration}

Following the previous framework in (Rahni et al., 2011), ${ }^{4}$ at each discrete time-point $k$, respiratory motion is defined by change of the current configuration of the organs at that time point, $\mathbf{c}_{k}$, from the configuration at a reference time point $\tau, \mathbf{c}_{\tau}$. As respiratory deformation is assume to be affine for each organ, the vector $\mathbf{c}_{k}$ thus consists of affine transformation parameters, which describe the deformation of points $\left\{\mathbf{p}_{k}^{i, o}\right\}_{i=1}^{N_{o}}$, where there are $N_{o}$ voxels for the organ $o$, as:

$$
\mathbf{p}_{\tau}^{i, o}=F_{k}^{o} \mathbf{p}_{k}^{i, o}+\mathbf{T}_{k}^{o},
$$

with $\tau$ being a reference time frame. However, in this form, as the points $\mathbf{p}_{k}^{i, o}$ are relative to a common origin which does not lie at the centre of each organ, the translation vector $\mathbf{T}_{k}^{o}$ does not reflect the general displacement of points for each organ at time $k$ from the reference frame $\tau$. They are also affected by noise in the affine matrix $F_{k}^{o}$ and hence may reflect this noise more than the general displacement of organs. Therefore, for each organ, it is replaced by a translation vector, $\mathbf{T}_{k}^{\star o}$, which reflects affine transformation around a coordinate system centred on each organ. This modified translation is defined as:

$$
\mathbf{T}_{k}^{\star o}=\mathbf{T}_{k}^{o}+\left(F_{k}^{o}-I\right) \overline{\mathbf{p}}_{\tau}^{o},
$$

where $\overline{\mathbf{p}}_{\tau}^{o}$ is the centroid of organ $o$ at the reference frame $\tau$. The effect of using this modified translation vector for both sequences is shown in Fig. 1. In Figs. 1(b) and (d) the two respiratory cycles with periods of around 4 $\mathrm{s}$ can be seen much more clearly than in Figs. 1(a) and (c), reflecting the actual amount of general displacement and showing movement in a more synchronous manner.

\subsection{External Motion}

The actual application of the motion estimation framework proposes the use of stereo cameras to observe the external surface of the torso. Although physical surface markers are present in the 4D MRI dataset, only the three abdominal markers, in particular the medial marker, show significant movement that is distinguishable due to the data resolution. The other three thoracic markers show less movement and appears to be dominated by noise due to the large voxel dimension ( $5 \mathrm{~mm}$ in the AP direction). Because of this and to approximate a stereo camera that observes a significantly larger portion of the torso surface, a method of estimating the movement of the surface along radial lines was used to give information on external respiratory motion. Here, this method will be compared against the physical markers.

The radial lines are defined to start from an axis parallel to the z-axis, passing through the centroid of the torso. The lines are perpendicular to this axis and pass through the centroid of each physical marker. The radial lines and physical markers are shown in Fig. 2(a).

The intersection of the skin with the radial line is found by interpolation at a fixed, manually defined, threshold that finds this radial position to sub-voxel accuracy, as far as image quality permits. This threshold is set so that the intersection occurs at a region where the intensity gradient along the radial lines is approximately linear. This "threshold" method is compared against a gradient method that finds the maximum intensity gradient along the radial line using finite difference. Both methods interpolate image intensity along the radial line at a $1 \mathrm{~mm}$ interval. Another comparative method is also defined where the gradient is interpolated (designated as the "Int. Gradient" method) at a finer interval of $0.1 \mathrm{~mm}$. Fig. 2(b) illustrates the sampling of intensity along a radial line and the threshold method.

All three methods are compared to the projection of centres of the physical markers along the radial lines (designated as the "Markers" method). The radial movement found for the medial abdominal marker, which shows the most movement, using all methods, are shown in Fig. 3(b).

The results show that all methods apart from "threshold" suffer from some form of quantisation due to the large voxel size in comparison to motion present. With these results, the "threshold" method is then used to find radial movement at virtual markers arranged in an $11 \times 19$ grid located between the two groups of physical markers (abdominal and thoracic) shown in Fig. 3(b). The radial positions of the skin at time $k$ then becomes the element of an observation vector $\mathbf{z}_{k}$, to be used in the estimation framework. 


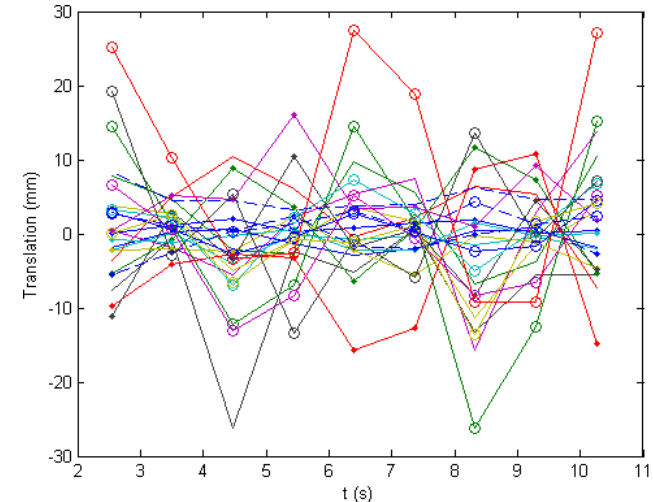

(a) Original absolute translation (sequence 1).

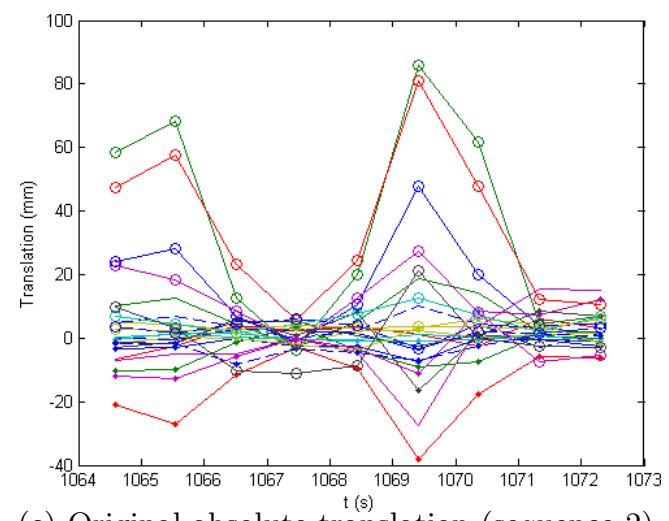

(c) Original absolute translation (sequence 2).

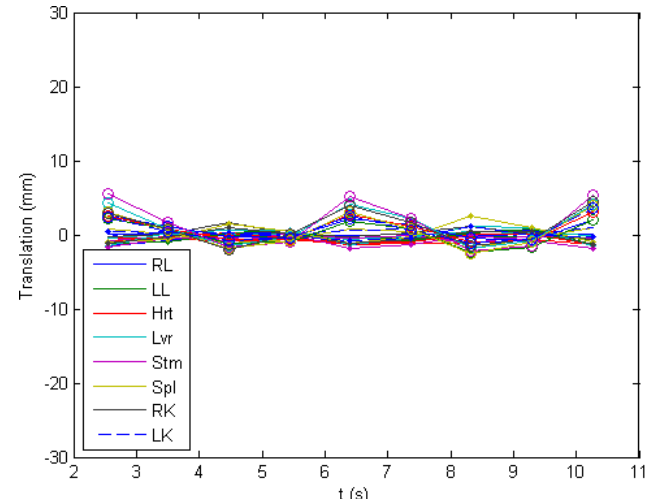

(b) Absolute translation from shifted coordinates (sequence 1 ).

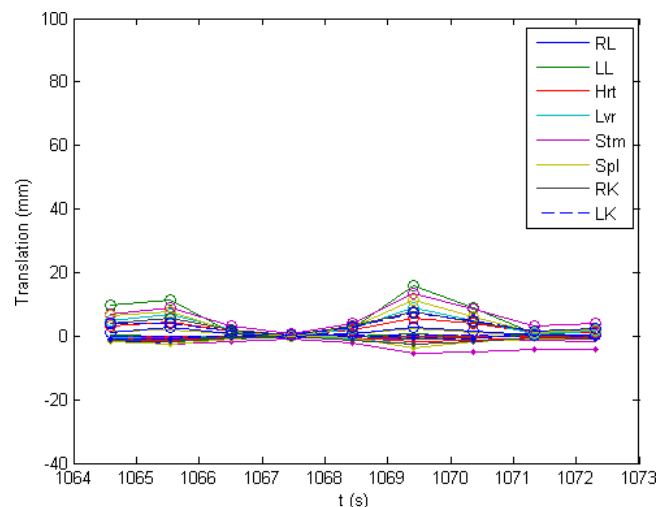

(d) Absolute translation from shifted coordinates (sequence 2 ).

Figure 1. Absolute translation from equations 1 and 2. The lines are according to the translation element: $T_{x}:$ solid, $T_{y}$ : with dots $(\bullet)$ and $T_{z}$ : with circles (०). The labels for the chosen organs are: RL: right lung, LL: left lung, Hrt: heart, Lvr: liver, Stm: Stomach, Spl: spleen, RK: right kidney and LK: left kidney.

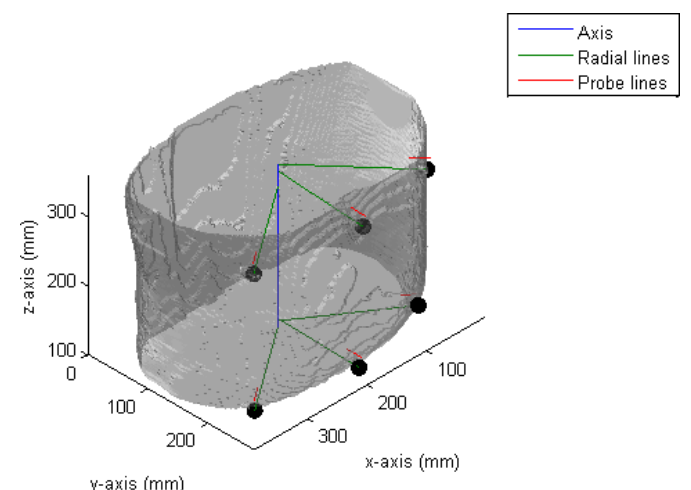

(a) Translucent surface renders with central axis and radial lines.

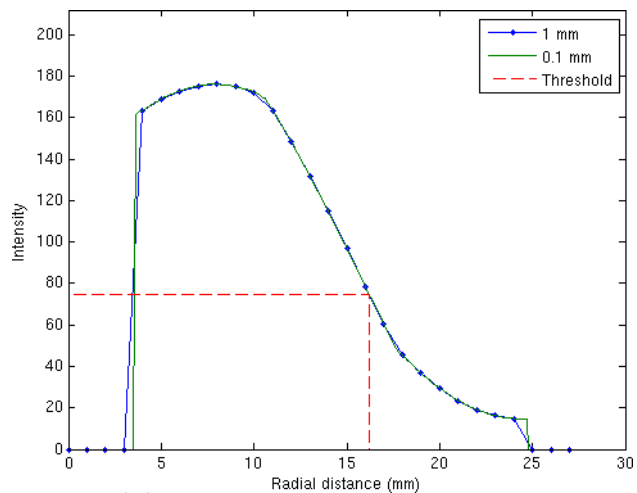

(b) Intensity along radial line

Figure 2. Fig. (a) shows the central axis and probe lines in relation to the torso surface. The physical markers are shown as black circles $(\bullet)$. Figs. (b) shows the intensity interpolated with two sampling intervals and the estimated radial position of the surface (red). 


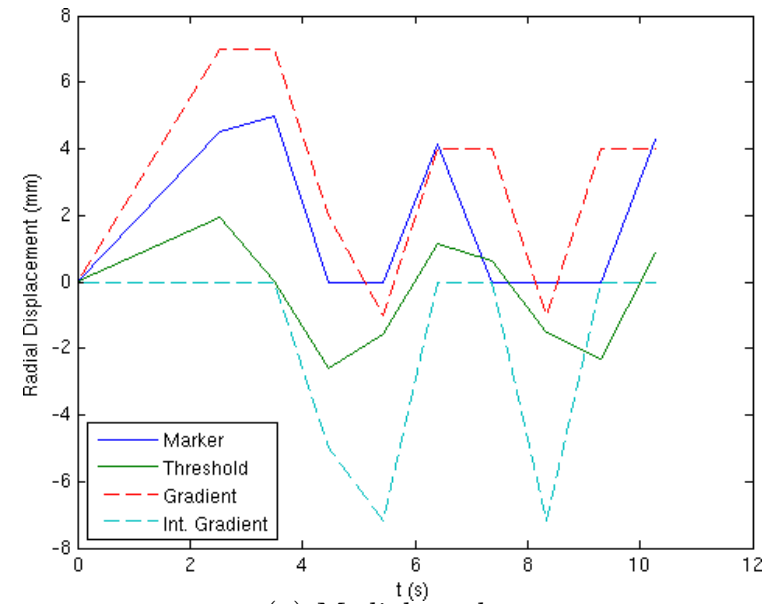

(a) Medial marker

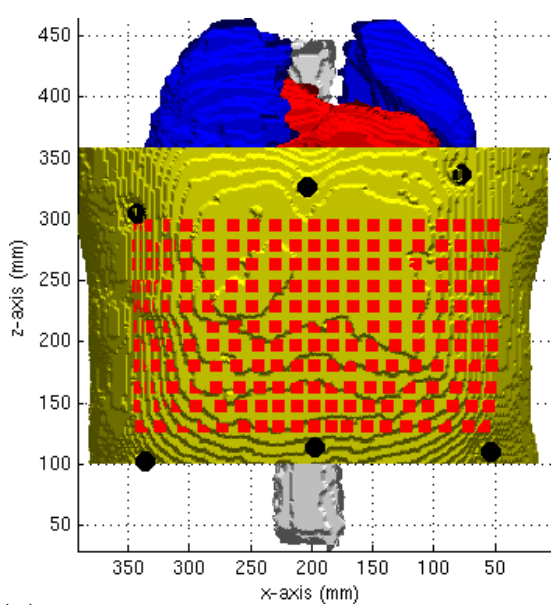

(b) Translucent surface renders with central axis and radial lines.

Figure 3. Figs. (a) shows the estimated radial displacement of the medial abdominal marker using different methods. Fig. (b) Virtual markers (red squares) shown together renders of segmented organs from (Rahni et al., 2013$){ }^{6}$

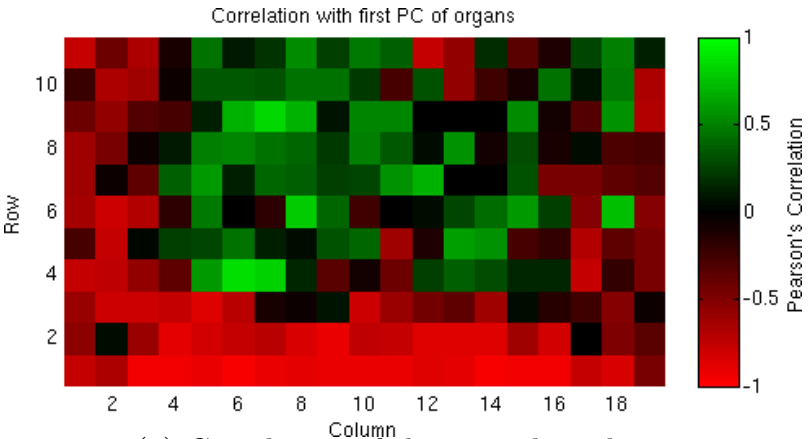

(a) Correlation of the virtual markers.

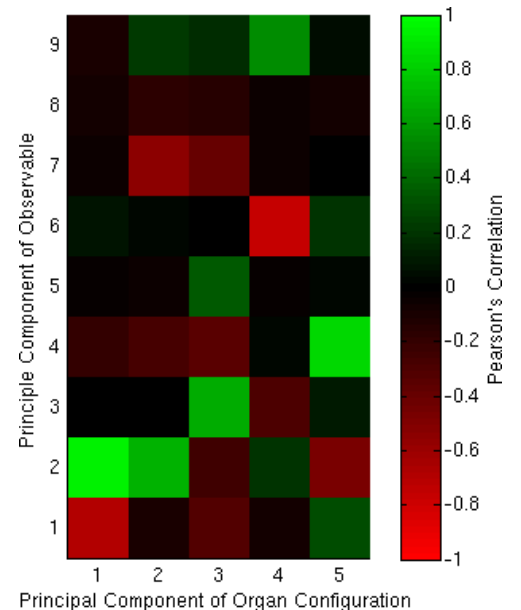

(b) Correlation between PCs of the observable and configurations.

Figure 4. Fig. (a) shows the correlation between the surrogates at the virtual markers and the first PC of organ configurations. Columns and rows refer to the grid of virtual markers in Fig. 3(b). Fig. (b) shows the correlation between PCs of the observable and configurations.

\subsection{External-Internal Motion Correspondence}

Following the estimation framework in previous work, ${ }^{4}$ principal component analysis is performed so that both organ configuration, $\mathbf{c}_{k}$, and the observable, $\mathbf{z}_{k}$, can be expressed in a lower number of dimensions. In this paper, the principal component that best reflect respiratory motion is used to simplify the analysis. In the estimation framework, all principal components with non-zero eigenvalues are used, as those with smaller eigenvalues were found to represent a considerable proportion of variation in both configuration, $\mathbf{c}_{k}$ and observation, $\mathbf{z}_{k}$.

\subsubsection{External-Internal Motion Correlation}

Fig. 4(a) shows the value of Pearson's correlation between the surrogates at the virtual markers (Fig. 3) and the first PC of modified organ configurations, while Fig. 4(b) shows the correlation between PCs of the observable and configurations. 


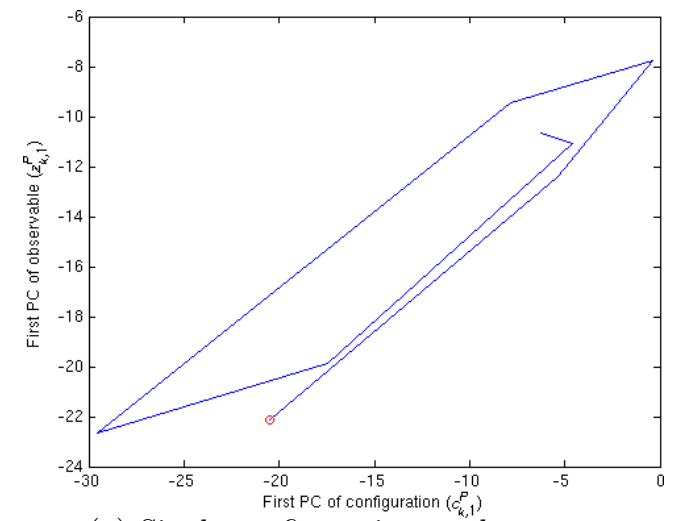

(a) Single configuration as the state.

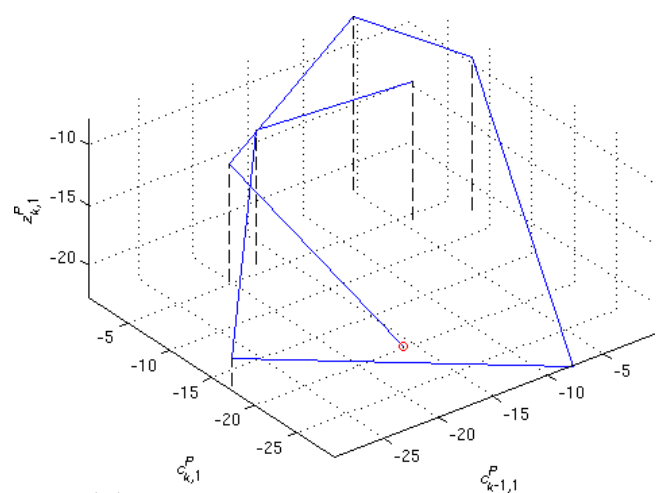

(b) Dual configuration as the state.

Figure 5. Trajectory to be modelled when the state is (a): a single organ configuration, and (b): two consecutive organ configuration. The plots show the joint trajectory for the first respective PCA projection of the configuration, $c_{k}^{P_{1}}$, and observable, $z_{k}^{P_{1}}$.

From Fig. 4(a) it can be seen that most of the virtual markers with a high absolute value of correlation lie in the abdominal region (smaller row number), although there are also some markers in the more superior rows with high absolute value of correlation. This thus relates to the observation that the amplitude of radial motion decreases as one moves away from the central abdominal physical marker in Fig. 3(b), thus the motion is less well defined. Fig. 4(b) shows that the second PC of the observable has the highest absolute value of correlation with the first PC of organ configurations (above 0.9 ). There are also high absolute values of correlation between the other PCs. However, a multidimensional regression model would account for these correlations when the PCs themselves are used as the regressor and regressand.

\subsubsection{Hysteresis}

It was also found that there is a noticeable amount of hysteresis, ${ }^{7}$ where the configuration, $\mathbf{c}_{k}$, does not vary in time with the observation, $\mathbf{z}_{k}$. Because of this, following a previous study, ${ }^{8}$ the state of internal motion that is to be estimated, $\mathbf{x}_{k}$, is a concatenation of organ configuration from two consecutive time points, $\mathbf{x}_{k}=\left[\mathbf{c}_{k}^{\mathrm{T}}, \mathbf{c}_{k-1}^{\mathrm{T}}\right]^{\mathrm{T}}$. When only the first principal component of configuration, $c_{k}^{P}$, and observation, $z_{k}^{P}$, respectively, are considered, then the state can be defined as $\mathbf{x}_{k}=\left[c_{k}^{P}, c_{k-1}^{P}\right]^{\mathrm{T}}$. The effect of having this augmented state variable as opposed to equating the state with configuration, i.e. $x_{k}=c_{k}^{P}$, is shown in Fig. 5 .

In Fig. 5(a), when the state to be estimated is equated to organ configuration, the trajectory to be modelled shows noticeable hysteresis. For most of the respiratory cycle, there are two values of $z_{k}^{P_{1}}$ for each value of $x_{k}=c_{k}^{P}$. However, in Fig. 5(b), when the state to be estimated consists of two consecutive organ configurations, there is a specific value of $z_{k}^{P_{1}}$ for each value of the augmented state,. The inhalation and exhalation portions of the trajectory to be modelled are also separated by the extra dimension that is introduced.

\section{CONCLUSION}

In this paper, representations of internal and external respiratory motion were defined. For internal motion, the use of modified translation vectors in addition to affine transformation matrices better reflects the general displacement of organs. External respiratory motion was also found to subvoxel accuracy, although the method used is only due to the data in hand. The correlation of virtual markers with internal motion varies and is seen better by the correlation between their respective PCA spaces. The correspondence of internal and external motion also shows hysteresis, in accordance with previous studies. ${ }^{9}$ Additionally, an augmented state vector was also shown to alleviate the phenomena of hysteresis in respiratory motion. These results will be a guide for further analysis with larger datasets and inform the approach for better estimation of internal motion from the torso surface. 


\section{Acknowledgement}

Ashrani Aizzuddin Abd. Rahni was funded by the Malaysian Ministry of Higher Education and University Kebangsaan Malaysia.

\section{REFERENCES}

[1] S. Nehmeh and Y. Erdi, "Respiratory Motion in Positron Emission Tomography/Computed Tomography: A Review," Semin Nucl Med 38(3), pp. 167-176, 2008.

[2] S. Chun, T. Reese, J. Ouyang, B. Guerin, C. Catana, X. Zhu, N. Alpert, and G. E. Fakhri, "MRI-Based Nonrigid Motion Correction in Simultaneous PET/MRI," J Nucl Med 53(8), pp. 1284-1291, 2012.

[3] C. Liu, A. Alessio, and P. Kinahan, "Respiratory motion correction for quantitative PET/CT using all detected events with internal-external motion correlation," Med Phys 38(5), pp. 2715-2723, 2011.

[4] A. A. Abd. Rahni, E. Lewis, M. J. Guy, B. Goswami, and K. Wells, "A Particle Filter Approach to Respiratory Motion Estimation in Nuclear Medicine Imaging," IEEE Trans Nuc Sci 58, pp. 2276-2285, Oct 2011.

[5] J. Jones, E. Lewis, M. Guy, and K. Wells, "A virtual dissection based registration to model patient-specific respiratory motion," in IEEE NSS/MIC Conf Rec, pp. 3571-3576, 2009.

[6] A. A. Abd. Rahni, E. Lewis, and K. Wells, "Extracting respiratory motion from 4d mri using organ-wise registration," SPIE Medical Imaging: Image Processing 8669, 2013.

[7] J. Escolar and A. Escolar, "Lung hysteresis: a morphological view," Histol Hispathol 19, pp. 159-166, 2004.

[8] D. Ruan, J. Fessler, J. Balter, R. Berbeco, S. Nishioka, and H. Shirato, "Inference of hysteretic respiratory tumor motion from external surrogates: a state augmentation approach," Phys Med Biol 53(11), pp. 29232936, 2008.

[9] P. J. Keall, G. S. Mageras, J. M. Balter, R. S. Emery, K. M. Forster, S. B. Jiang, J. M. Kapatoes, D. A. Low, M. J. Murphy, B. R. Murray, C. R. Ramsey, M. B. van Herk, S. S. Vedam, J. W. Wong, and E. Yorke, "The management of respiratory motion in radiation oncology report of AAPM Task Group 76," tech. rep., American Association of Physicists in Medicine, 2006. 